\title{
SOME EXPERIMENTS ON THE FUNCTION OF THE PERICARDIAL ORGANS IN CRUSTACEA
}

\author{
By J. S. Alexandrowicz and D. B. Carlisle \\ The Plymouth Laboratory
}

(Text-figs. I-II)

The experiments reported here were designed to test the assumption that the nervous organs found in the pericardial cavity of the Crustacea, and termed 'pericardial organs'1 (Alexandrowicz, I953 $a, b$ ), have a secretory function. Their situation suggested that the substances liberated by them into the blood of the pericardial cavity probably act on the heart. Accordingly, the aim of this series of experiments was to determine what might be the effect of these postulated hormones on the crustacean heart.

\section{METHOD}

The method employed consisted in preparing an extract of the pericardial organs and testing its action by adding it to the fluid perfusing the isolated heart.

The observations were chiefly made with the two species of large crabs easily available at Plymouth, Cancer pagurus and Maia squinado. The lobster (Homarus vulgaris) proved to be less suitable experimentally. Some experiments were performed at Naples ${ }^{2}$ with a stomatopod, Squilla mantis. There were perfused in all I5 hearts of Cancer, 26 of Maia, 4 of Homarus and Io of Squilla.

As pointed out in the paper quoted above, the pericardial organs can be found most easily in the pericardial cavity of crabs, where they lie at the openings of the branchio-cardiac veins. With Maia there is a chance of removing all the elements of the organs of one side hanging together, i.e. the

${ }^{1}$ R. I. Smith in a figure illustrating the course of the cardiac nerves in Cancer irroratus (Biol. Bull. Woods Hole, 1947, Vol. 93, p. 77) has shown nerves designated as 'lateral pericardial plexus', which are certainly the elements of the pericardial organs. This 'lateral pericardial plexus' was also observed in Pugettia producta by J. P. Heath (Fourn. Morph., I94I, Vol. 69, p. 48I). The term 'pericardial organs', proposed by one of the present writers (J. S. A.) in his previous publication, and adopted without knowledge of the designation previously given, seems more appropriate in view of the unusual structure and function of these elements: this term will also embrace the analogous elements in stomatopods first described as parts of the 'system of dorsal trunks'.

${ }^{2}$ We wish to express our gratitude to Prof. R. Dohrn for the facilities offered to us. One of us (D. B. C.) is indebted to the Browne Fund of the Royal Society for financial assistance, which made his visit to Naples possible. 
anterior and posterior bars with their prolongations and the longitudinal trunks. In Cancer the organs must be taken out in two portions separately, i.e. the anterior and posterior bars each with the adjoining parts. This procedure is also often more convenient in Maia since it is easier, and since in the preparation of extracts the organ need not be in one piece.

The isolated organs were first rinsed in the saline used for perfusion and then triturated with sand in a little of the same solution. The suspension was filtered and the residue washed with several more portions of fluid totalling Io $\mathrm{ml}$. The filtrate was used, in most of the experiments, as the stock solution from which certain amounts were taken and added to the perfusion fluid. The stock solution was prepared fresh before each series of tests.

In Homarus only a part of the pericardial organs is arranged in discrete trunks: the other part is spread as plexuses over the wall of the pericardium. Moreover, even the trunks themselves, which are much shorter than in crabs, cannot be easily distinguished from other tissues. As the dissection is difficult and its result uncertain only a few trials were made.

In Squilla mantis the pericardial organs are present in the Ist-5th abdominal segments and are of two kinds. Those of one kind, short trunks flattened to lamellae spanning the cleft between the dorsal muscles, can be easily found. To expose them, parts of the terga have to be removed, care being taken not to tear away the tissues in the mid-line. The lamellae are recognizable by their shape and position, sometimes also by their slightly greyish colour differing from the yellowish hue of the muscles. The elements of second kind, spreading over the pericardial wall, are in practice scarcely distinguishable in unstained preparations.

The saline used for perfusion in all four species was prepared according to the formula of Welsh (I939a) (modified Pantin's solution). The perfusion of the heart of the decapod crustaceans was performed according to the method used by Welsh (I939a) with some minor modifications of the apparatus and procedure.

The routine was as follows.

(i) The legs were cut off and the dorsal part of the carapace and of the pericardium removed.

(ii) The pericardial organs were exposed by cutting the lateral heart ligaments. The organs were excised and the extracts prepared.

(iii) The heart, with parts of the arteries, was cut out and put into a flat dish with perfusion fluid to rinse. Sea water may also be used for rinsing the heart, as it seems not to affect its later behaviour.

(iv) A cannula already fixed into the rubber stopper was introduced into the heart through the sternal artery. A cannula with a spherical enlargement of the tip may meet resistance at the arterial valve, and only when it can be pushed freely farther into the heart cavity is there a certainty that the valve has been passed. 
(v) A ligature was tied round the sternal artery to fix the cannula in place, and a second one round the anterior aorta.

(vi) The heart was put into a short glass tube with a capacity of $80 \mathrm{ml}$. , the stopper fixed in place, the tube filled with perfusion fluid, and the thread of the anterior ligature attached to the writing lever.
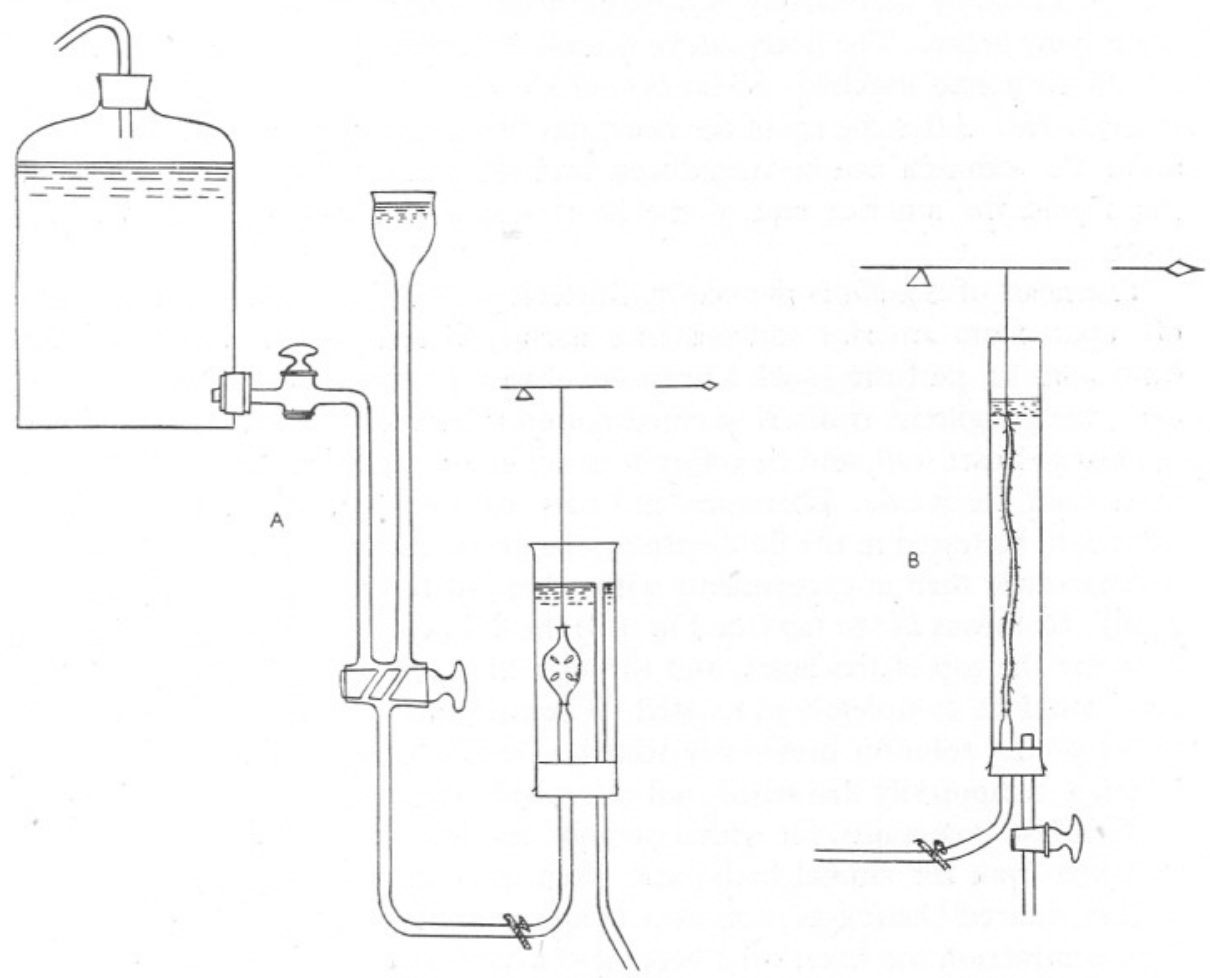

Fig. I. A, the apparatus used for perfusing the hearts of Cancer, Maia and Homarus. The Mariotte bottle was only used for prolonged washings. Before, during and for a short time after each test perfusion was made from the thistle funnel, with constant addition of fluid. The rate of perfusion was controlled by means of the screw clamp. B, the modification of the heart chamber used for perfusing the heart of Squilla, drawn to a larger scale than A.

(vii) Perfusion was begun first from the Mariotte bottle (Fig. IA), but, as differences in the level of the perfusion fluid may cause some changes in the heart rhythm, the two-way stopcock was turned before each test and the perfusion was made for some time from the thistle funnel into which later was poured the saline with the substance to be tested. In this way the same conditions of perfusion prevailed before, during and after each test. The Mariotte bottle was only used for prolonged periods of unattended perfusion and for washing out test solutions. 
The volume of each test solution, i.e. saline with the substance to be tested added, was as a rule the same, viz. $20 \mathrm{ml}$.

To expose the heart of Squilla mantis the dorsal parts of the terga and of the extensor muscles must be removed as far as is necessary to give free access to the long heart tube. The latter can then be taken out, preferably from the hind end forwards, by successively cutting the arteries and the connexions with the underlying organs. The heart can be completely isolated, but it is safer to leave it with the gonad attached. So far as could be observed this does not influence its reactions. After the tip of the heart has been cut off close to the posterior aorta, the cannula can be introduced and secured by a ligature. A thread put round the anterior end of the heart serves to attach it to the writing lever.

The heart of Squilla is pierced by thirteen pairs of ostial orifices and sends off, apart from anterior and posterior aortae, fifteen pairs of arteries. The conditions for perfusing such a heart are obviously very unfavourable. Moreover, the ganglionic trunk is situated not on the inside but on the outside of the dorsal heart wall, and therefore it is not easily accessible to the perfusing fluid from the inside. Therefore, in order not to dilute too much the substances to be tested in the fluid bathing the heart, the tube used for perfusion was narrower than in experiments with other crustaceans and contained only $20 \mathrm{ml}$. By means of the tap (see Fig. I B) the fluid could be kept at a level just covering the top of the heart, and through the same tap the contents of the tube could be completely evacuated. When refilled the tube contained only traces of the solution previously tested. During change of fluid the heart action is temporarily disturbed, but afterwards resumes normally.

With few exceptions, for which no good reason could be found, the heart, removed from the animal body, and often quite motionless during the dissection, started beating as soon as it was immersed and perfusion began. At each contraction the heart tube becomes twisted so that its top moves clockwise round the vertical axis. This movement is obviously due to the spiral course of the muscle fibres; the vertical component of the contractions, however, has sufficient amplitude to give quite satisfactory records.

\section{Action of Extracts of the Pericardial Organs and of Drugs}

\section{Cancer pagurus}

\section{Action of Pericardial Organ Extracts}

The addition of extract of its own pericardial organs to the perfusion fluid has a very distinct effect on the heart beat of Cancer, increasing the frequency and amplitude of the contractions. This effect proved to be dependent on the activity of the extracts and on the sensitivity of the heart, both factors being subject to individual variations. The record in Fig. $2 \mathrm{~A}$ was obtained with a 
concentration of $0.4 \mathrm{ml}$. of the stock solution diluted to $20 \mathrm{ml}$. On occasion a distinct reaction was noted with $0.1 \mathrm{ml}$. of the stock solution of the extract, i.e. using $\mathrm{I} / \mathrm{IOO}$ of the active substance of the organs of one animal, diluted in

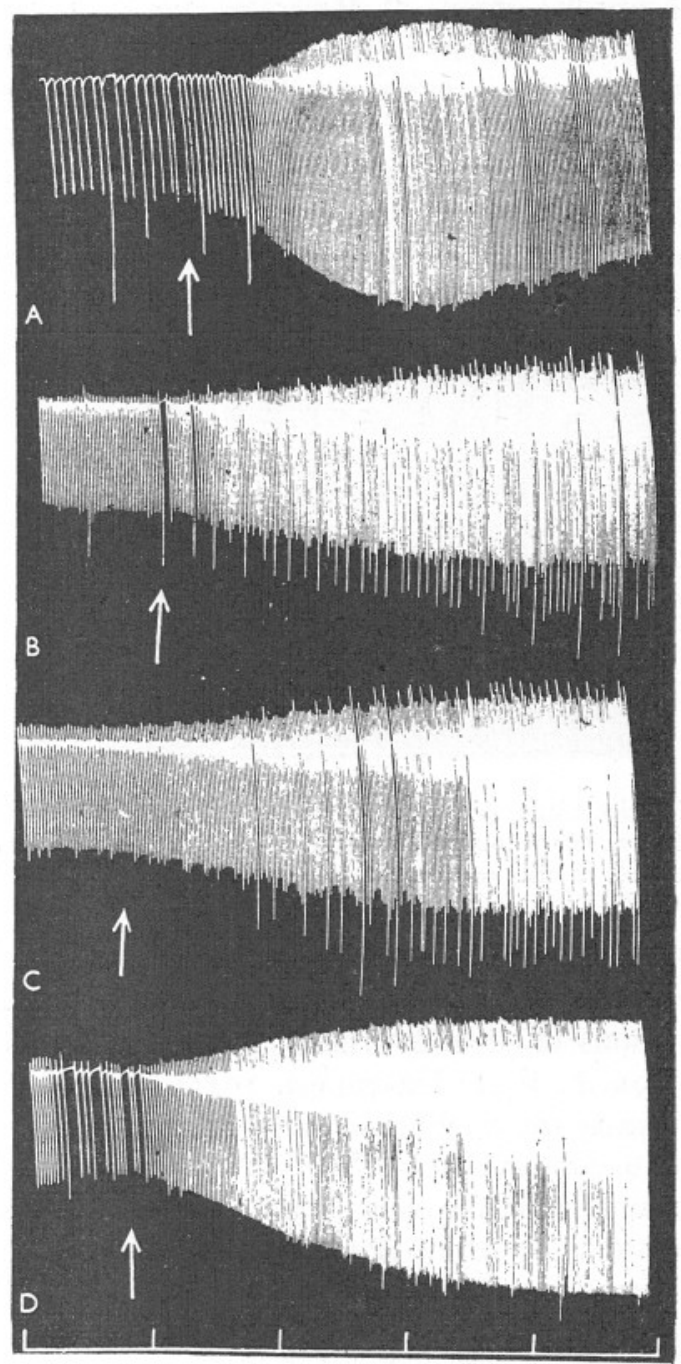

Fig. 2. The successive action on the same heart of Cancer of (A) pericardial organ extract of Cancer, (B) adrenaline ( $\left.\mathrm{I}: \mathrm{IO}^{6}\right)$, (C) noradrenaline $\left(\mathrm{I}: \mathrm{IO}^{6}\right)$ and (D) the same extract as in A. The records read from left to right; the arrows indicate approximately the time at which the reagents reached the heart; time scale in minutes.

$20 \mathrm{ml}$. of saline. In other experiments with other hearts and extracts greater quantities, up to $4 \mathrm{ml}$. of the stock solution, were needed to produce a similar reaction. 
The effect of the extract in exciting the heart beat is not long-lasting and after all the fluid containing the extract has passed through the heart and been replaced by ordinary saline the heart gradually returns to its previous rhythm.

Extracts of pericardial organs of Maia squinado (Fig. 3) and of Portunus puber produced similar reactions in the Cancer heart. To obtain effects equal to the above with the Maia extracts a higher average dose was required, indicating that in the organs of this animal there is less accelerating principle. With Portunus comparisons are uncertain, not only because of its much smaller size, but also because the dissection of its pericardial organs is difficult and probably loses much of their parts.

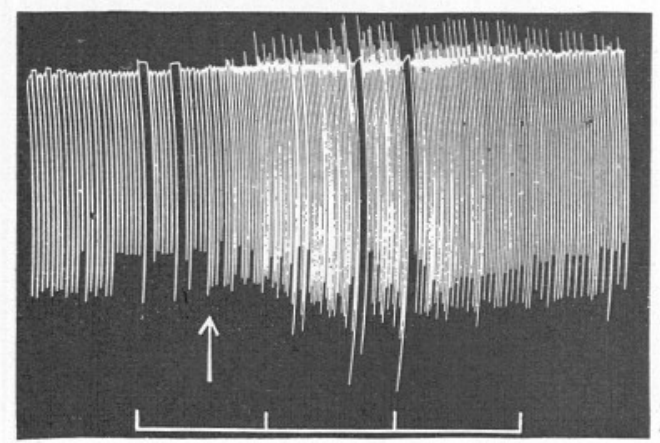

Fig. 3. The effect of pericardial organ extract of Maia on the heart of Cancer. The record reads from left to right; the arrow indicates approximately the time at which the extract reached the heart; time scale in minutes.

\section{Action of Adrenaline, Noradrenaline and Tyramine}

The action of adrenaline and acetylcholine on crustacean hearts is known through investigations of several writers (Hogben \& Hobson, I924; Bain, I929; Welsh, I939 $a, b$; I942; Davenport, I94I; Ghiretti, I947), and there was no need to control the established facts. We tried these drugs, however, in order to be able to compare their effects with those produced by the extracts of the pericardial organs on the same hearts and under the same conditions.

Of the drugs tested, adrenaline (Parke, Davis and Co.) and noradrenaline (Levophed, of Bayer), at concentrations of $1: 10^{6}$ or $1: 10^{7}$, showed effects most resembling those of the extracts. These two drugs and the extracts were so much alike in their effect-an acceleration of the beat and a gradual increase in amplitude, sometimes to remarkable maxima, followed by an equally gradual return to normal-that often no differences could be noticed. However, some hearts appeared to react somewhat differently in response to the extracts from their reaction to the drugs. Fig. 2 shows records obtained with the same heart treated (A) with the extract, then (B) with adrenaline, (C) with noradrenaline and (D) again with the same extract, the last trial to eliminate 
the possibility of the previous treatment having influenced the response of the heart to that following. It will be noticed that the extract, apart from the increase in frequency and amplitude, has also a steadying effect on the beat, indicated by more even contractions.

The action of acetylcholine is different (Fig. 4). It accelerates the heart rhythm and may also increase slightly its amplitude; at higher concentrations, however, the amplitude not only does not become greater, but markedly decreases, becoming even smaller than during the previous 'normal' beat till the heart stops in diastole or nearly in this condition. On the latter point our observations on Cancer and Maia differ from those of Welsh (I939a, b), Davenport (I94I) and Ghiretti (I947), who recorded stoppage not in diastole but in systole. In conversation with Dr J. H. Welsh we have learned that in his experiments he used a counterbalanced writing lever, whereas in the experiments reported in this paper we used a spring-loaded lever, so that the heart was always pulling against the tension of a spring. This may explain the different conditions of stoppage after acetylcholine in the two sets of experiments.

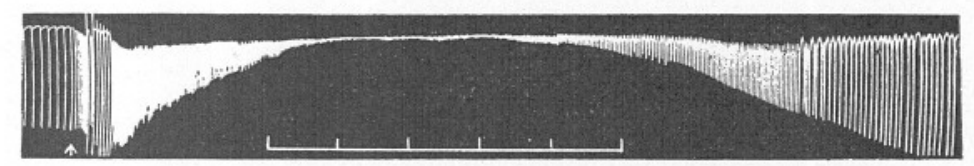

Fig. 4. The effect of acetylcholine $\left(1: \mathrm{IO}^{3}\right)$ on the heart of Cancer. As in all the kymograph records reproduced here, contractions are downwards so that the stoppage of the heart has taken place in diastole. The record reads from left to right; the arrow indicates approximately the time at which the drug reached the heart; time scale in minutes.

The experiments with tyramine-hydrochloride (L. Light and Co.) gave uncertain results. In some a slowing action of this drug at concentrations of $I: 10^{7}, I: 10^{6}$ or $I: 10^{5}$ was recorded; in others it was ineffective at concentrations of $I: I O^{6}$ and slightly accelerating at $I: I O^{5}$. Since the effects obtained do not resemble those of the extracts, this substance is not likely to prove the active principle of the organs in question.

The assumption that the active principle may be adrenaline-like was corroborated by the fact that pericardial-organ extract from Cancer, after addition of sodium hydroxide, gave a distinct fluorescence reaction of the same green colour as adrenaline. The intensity of the reaction shown by the extracts of organs of a single specimen prepared with $\mathrm{IO} \mathrm{ml}$. saline corresponded approximately to a $\mathrm{I}: \mathrm{IO}^{6}$ solution of adrenaline. On the assumption that this principle would be released by the organs into the pericardial cavity, we tested blood taken from this cavity and found it to give a fluorescence of the same kind. This reaction, however, was not shown by blood of the same animal taken from the leg arteries. The extracts of the organs and the blood of Maia 
either did not give the fluorescence reaction at all or at the most very slightly. The extracts from the organs of Portunus gave a distinct positive fluorescence reaction.

It follows from these experiments that the pericardial organs in decapod crustaceans release some adrenaline-like principle which has an excitatory effect on the heart beat. Experiments with another crab, Maia squinado, show, however, that this is not a complete statement of the action of these organs.

\section{Maia squinado}

\section{Action of Pericardial Organ Extracts}

The action of the pericardial-organ extract on the heart of Maia is strikingly different from that in Cancer for it decreases the frequency of the

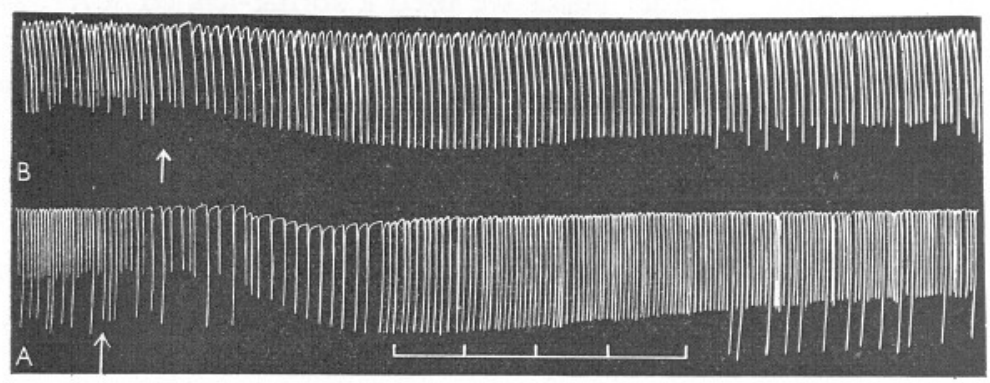

Fig. 5. The effect on the heart of Maia of its own pericardial organ extract. A shows the fourfold action-decrease in frequency and increase in amplitude of the beat, increase in tonus and the greater regularity of the beat, with a return to its previous condition as the extract is washed out of the heart; B shows more clearly the steadying effect of the extract, and in this heart there was no increase in tonus. The records read from left to right; the arrows indicate approximately the time at which the extracts reached the hearts; time scale in minutes.

beat. The amplitude becomes only a little greater and never reaches such a height as in Cancer, and the higher concentrations do not bring about a further increase.

Other effects, not always noticeable, are the steadying of the beat and an increase in tonus. The fourfold action of the extracts may be seen in Fig. $5 \mathrm{~A}$. The steadying effect is also well illustrated by Fig. 5 B showing how the uneven beat of the heart became more regular after treatment with the extract and, after cessation of the action of the extract, returned to its previous rhythm with the same irregularities.

The heart in Maia proved to be less sensitive than in Cancer, and for recording the action of the extracts $2-5 \mathrm{ml}$. of the stock solution were made up to $20 \mathrm{ml}$. of the perfusion fluid.

The result of the perfusion of Maia heart with the extract of Cancer is 
shown in Fig. 6. The heart responded by an increase in amplitude and by a slight decrease in frequency of beat. This shows, therefore, that the hearts of the two species react differently from each other to their own respective pericardial extracts, and these differences are maintained when the hearts are subjected to treatment with extracts from the opposite species.

\section{Action of Drugs}

The heart of Maia responds to adrenaline by an increase in frequency and amplitude of beat. It will, however, be noted that Maia heart is less sensitive than Cancer heart to this principle in reacting with less vigorous contractions; the effect shown in Fig. 7 is rather exceptional and different sensitivities of the hearts could often be observed. Thus the reaction in one heart was just

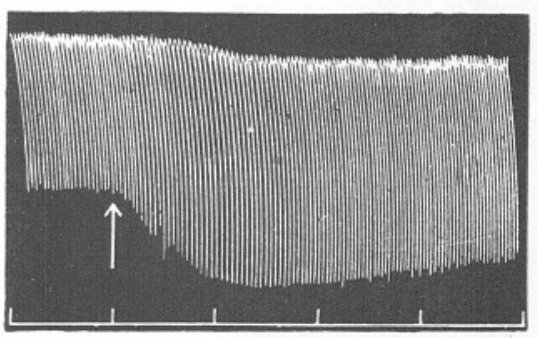

Fig. 6.

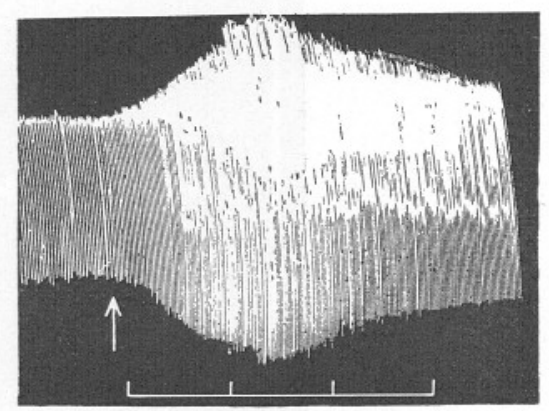

Fig. 7 .

The records read from left to right; the arrow indicates approximately the time at which the active principle reached the heart; time scale in minutes.

Fig. 6. The effect of pericardial organ extract of Cancer on the heart of Maia.

Fig. 7. The effect of adrenaline $\left(1: 10^{6}\right)$ on the same heart of Maia.

distinct with a concentration of $\mathrm{I}: \mathrm{IO}^{8}$, whereas in another no effect was noted with this concentration. The action of noradrenaline is similar to that of adrenaline, but sometimes somewhat stronger contractions were observed after treatment with noradrenaline than with adrenaline of the same concentration.

The action of acetylcholine does not show marked differences from that on the heart of Cancer.

Tyramine in higher concentrations, such as $\mathrm{I}: \mathrm{IO}^{5}$, first slows the beat and decreases the amplitude and then accelerates the beat and increases the amplitude. In lower concentrations, such as $\mathrm{I}: \mathrm{IO}^{7}$, a slowing of the beat was recorded. The regular heart rhythm seems to be affected by this drug and occasionally the heart stops for a while. Tyramine thus seems unable to play the main role in the action of the pericardial organs. 


\section{Homarus}

Because of the arrangement of the pericardial organs in Homarus only a small part of them could be cut out, and the extracts contained, at best, only a small fraction of the active substance presumably present in the whole of the organs. Applied to the heart of Homarus, which was perfused in the same way

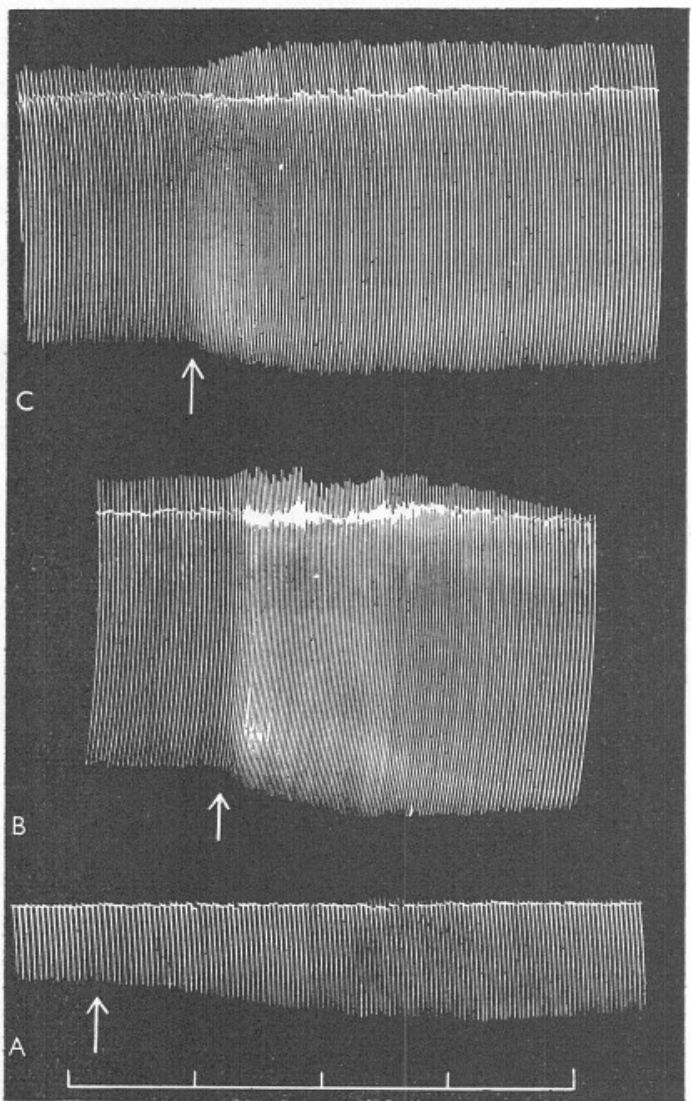

Fig. 8. The effect on the heart of Homarus of (A) pericardial organ extract of Homarus, (B) pericardial organ extract of Cancer, and (C) pericardial organ extract of Maia. Records read from left to right; the arrows indicate approximately the time at which the extracts reached the heart; time scale in minutes.

as those of crabs, the extracts increased the frequency and amplitude of beat (Fig. 8A); both effects however being less pronounced than in Cancer. Extracts from Maia and from Cancer both have an excitatory effect and no marked difference in the response to either was noticed (Fig. 8B, C). Even when a certain Homarus heart showed a peculiar reaction by first increasing the frequency and then slowing it abruptly the records were practically identical with Maia and with Cancer extracts. In general the heart of the lobster 
appeared to be less sensitive to the extracts and to adrenaline and noradrenaline as well. The threshold concentration of both these compounds was as high as $\mathrm{I}: \mathrm{IO}^{5}$.

\section{Squilla mantis}

Obviously, owing to its anatomical features, only a part of the inside of the heart of Squilla can come into direct contact with the undiluted stream of perfusion fluid, and therefore, if some substances are added to this fluid, they can act on remaining parts of the heart elements only after being diluted by the fluid already filling the container. Therefore even negative results would not be decisive. Fortunately, it was found that extract made from the pericardial organs of a single specimen (five lamellae) was sufficient to cause a distinct increase in frequency and amplitude of beat (Fig. 9A). Similar effects were brought about by adrenaline and noradrenaline (Fig. 9B). The concentration of these substances in the perfusion fluid was $1: 10^{6}$, but, as just pointed out, the concentration at the moment of their action on the receptive elemerits of the heart was uncertain.

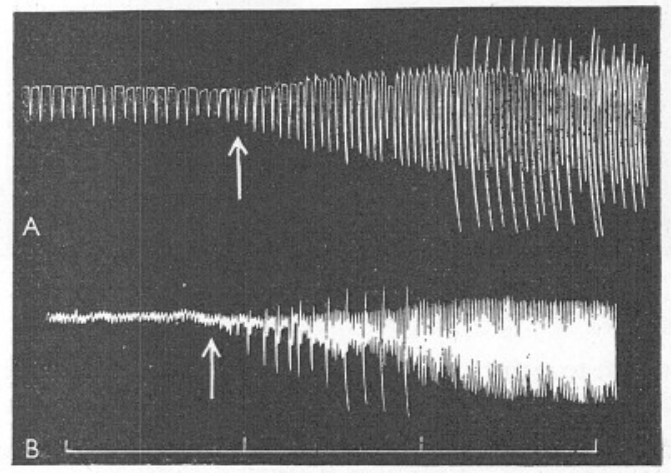

Fig. 9. The effect on the heart of Squilla of (A) pericardial organ extract of Squilla, and (B) adrenaline $\left(\mathrm{I}: \mathrm{I} \mathrm{O}^{6}\right)$. The records read from left to right; the arrows indicate approximately the time at which the reagents reached the heart; time scale in minutes.

The action of the extracts from crabs on Squilla heart, and vice versa, could not be determined, since all these animals were not available at one and the same place, and we had not yet worked out a reliable method of preserving the active extracts for future use.

The fluorescence test with the pericardial organs of Squilla gave uncertain results. At this stage of the work it could not be determined whether the active principle in Squilla is different from that in Cancer or too small in amount to give the reaction.

\section{Tests on Release of Secretion into the Blood Stream}

In order to test the supposition that the pericardial organs release the active substance into the blood the following experiments on crabs were performed. 
Perfusion of the heart with blood. Blood was taken, one sample from the leg arteries and another from the pericardial cavity of the same animal. Each sample was filtered and, after dilution with an equal amount of saline, tested on the heart. The result of this experiment in Cancer (Fig. Io) shows that the blood from the pericardial cavity has a similar stimulating effect to the extracts of the pericardial organs, whereas that from the leg arteries slows the heart beat and decreases its amplitude. ${ }^{1}$

Perfusion of the pericardial organs in situ. An animal was bled through the leg arteries and then from the pericardial cavity through a small hole bored into the carapace above the heart. After the animal had been fixed by means

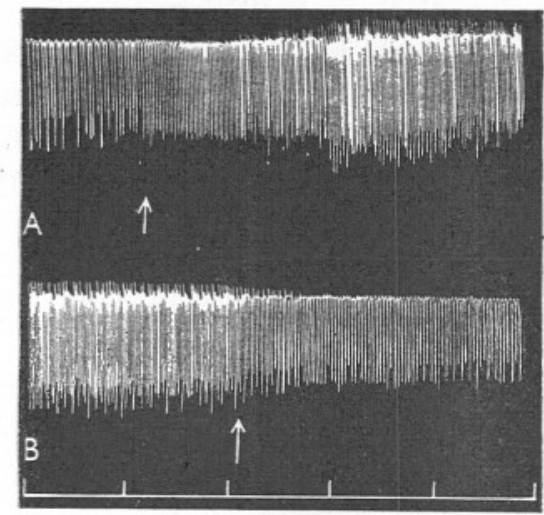

Fig. ro.

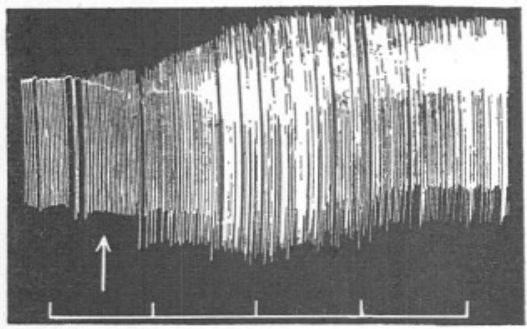

Fig. II.

The records read from left to right; the arrow indicates approximately the time at which the active material reached the heart; time scale in minutes.

Fig. I0. The effect of blood on the heart of Cancer. The blood was filtered after clotting and the filtrate diluted with an equal volume of saline. A, blood taken from the pericardial cavity stimulates the heart; B, blood taken from the leg arteries depresses it.

Fig. II. The effect on the heart of Cancer, of saline which had been perfused through the * pericardial cavity in situ, passing over the pericardial organs.

of a clamp with the ventral side upwards, the pericardium was perfused through three tubes introduced into the branchio-cardiac veins. The fluid flowing out through the hole in the carapace was collected and tested on the heart of a second animal perfused in the ordinary way. In experiments made with Cancer this fluid showed a distinct stimulating effect (Fig. II) and gave a positive fluorescence reaction.

With Maia the results were uncertain. As the active substance of the pericardial organs and the blood both slow the beat, the differences in their action

1 Blood of crustaceans taken out from the animal body ceases to be a proper medium for the tissues: it affects the heart action, decreasing the amplitude of the beat, and often causing an irregular rhythm and sometimes even stoppage of the heart. This is obviously due to the quickly occurring changes in the composition of the blood. 
(an increasing of amplitude by the former and decreasing by the blood) are hardly distinguishable. Moreover, the exactness of the experiments with perfusion in situ is somewhat impaired by the fact that, even after a rinsing of the pericardial cavity prior to collecting the fluid for the tests, some of the blood remains there and passes into the perfusion fluid, revealing its presence by an opalescence. The presence of blood may influence the reaction of the heart on test, but the preliminary washing of the pericardial cavity cannot be prolonged lest the pericardial organs should be exhausted.

\section{POSSIBLE ERRORS}

As noted by other workers in this field, allowance must be made for the hearts taken out of various specimens behaving in quite different ways. Some hearts start to contract strongly and in fairly regular rhythm immediately on being immersed in the saline and after the stream of perfusion fluid passes the cavity of the heart; others need some time to adjust themselves to these conditions. Several did not contract at all for a long time, but did so after addition to the perfusion fluid of a small amount of the pericardial extracts or of adrenaline, and then continued to beat regularly for several hours. The degree of stretching of the heart and the rate of perfusion must be found empirically. We tried at first, in order to compare the action of various substances under conditions as similar as possible, to maintain a constant rate of perfusion, viz. $20 \mathrm{ml}$. of the solution through the heart in $3 \mathrm{~min}$. This was approximately the rate of flow in most of the experiments, but various hearts show individual predilections, and even the same heart, after beating for some time at a certain speed of perfusion, may require later a faster one. Usually the hearts respond to the accelerated perfusion with stronger contractions. However, even this rule is not without exception: sometimes the heart worked definitely worse at the flow-rate usually used and needed one much slower.

This different behaviour may be partly due to blood clots forming in the heart and thus creating different conditions for passage of the perfusion fluid. It may be, too, that individual variations in the situation of the ganglionic trunk of the heart play some role. For instance, since this trunk lies on the inside of the dorsal heart wall and is partly covered by the muscle bundles, it is possible that, because of variations both in the thickness of these bundles and in the position of the ganglion cells, this pacemaker may be more or less accessible to the action of the mediators. The thickness of the muscle bundles is perhaps responsible for less satisfactory results with the hearts of the largest specimens of Maia. We preferred, therefore, to use medium-sized animals (carapace measuring about $\mathrm{I} 3 \times \mathrm{I} 7 \mathrm{~cm}$.), rather than large ones.

The results of the experiments depend not only on the variable sensitivity of the hearts but also on an even more unstable factor, the activity of extracts, which appear to vary greatly, presumably in relation to the condition of the animals. 
All the experiments recorded above were made during the summer months. When we tried to continue this work in January, however, the results obtained werè unexpectedly confusing. A concentration of Maia organ extracts several times higher than that always found previously to be effective did not produce any reaction and even in one specimen slightly accelerated the beat.

It was then found that the hearts responded to treatment with adrenaline and noradrenaline in a way never previously observed by us and, moreover, in successive experiments conducted under identical conditions the results obtained were very different. Thus one heart did not react at all to a concentration of I:5000; another reacted to a concentration of $\mathrm{I}: \mathrm{I0}, 000$ by a slight acceleration without an accompanying increase in amplitude; again another, treated with a concentration of $\mathrm{I}: \mathrm{I}, 000,000$, responded by a spectacular increase in tonus, acceleration, and a notable decrease in amplitude. This was the same heart which accelerated in response to a highly concentrated pericardial organ extract. The reason for this abnormal behaviour of the hearts was possibly the low temperature at which the animals were kept in the outdoor storage tanks.

Although the experimental evidence speaks in favour of a hormonal nature of the substance released by the pericardial organs it can be objected that the active substances might be compounds of the tissues constituting the organs or of blood which pass into the extracts. It is known from experiments of Welsh (I939a) that nervous tissue from both the central nervous system and from peripheral nerves has an effect on the heart, and, as this writer concluded, 'the active principle in question is, with little doubt, acetylcholine'. We tested the effects of an extract of leg nerves of Maia and found that these were the same as in Welsh's experiments, and certainly different from those produced by the extract of the pericardial organs. Pieces of pericardial wall and blood clots even much larger than those which often adhere to the pericardial organs do not produce any effect.

Since on dissection of the pericardial organs in Homarus the removal of a certain amount of connective tissue with the organs was unavoidable, extracts from pieces of this tissue were made and tested but did not produce any apparent effect.

\section{Discussion}

The recorded results favour the assumption that the pericardial organs of the Crustacea contain some specific substances influencing the heart action. It must be emphasized that very small amounts of these substances are able to produce distinct effects, as may be inferred from the following estimation. The weight of the pericardial organs in a large specimen of Maia (carapace I $7 \times 20 \mathrm{~cm}$., weight I560 g.) was found to be $5 \mathrm{mg}$. and in Cancer (carapace $2 \mathrm{I} \times \mathrm{I} 8 \mathrm{~cm}$., weight I8IO g.) $-8 \mathrm{mg}$. (Both organs were rinsed in saline and some of this fluid adhered to them during the weighing operation.) As stated 
before, I/IOO of the active substance of these organs diluted in $20 \mathrm{ml}$. was sufficient to show its action on the Cancer heart. Assuming that this substance made up as much as $5 \%$ of the total wet weight of the organs, which is certainly well on the high side, it is evidently efficient at a concentration of I : $5,000,000$ or less.

In Squilla the weight of pericardial organs was not measured directly but from the dimensions of a lamella (about I000 $\times 300 \times 50 \mu$ ) the weight of five of them can be supposed not to exceed $0 . \mathrm{I} \mathrm{mg.;} \mathrm{then} \mathrm{the} \mathrm{concentration} \mathrm{of} \mathrm{the}$ active substance in Io $\mathrm{ml}$. of the perfusion fluid assuming the same $5 \%$ of total wet weight would be $\mathrm{I}: 2,000,000$. As has been said above, this concentration was lowered by the fluid bathing the heart. Clearly then, in the investigations aiming at identifying the active principle, only such compounds as are capable of giving similar effects at very low concentrations ought to be taken into account.

There is one feature in the behaviour of the hearts in the two species of the Brachyura which requires particular consideration. Were the experiments performed with the heart of Cancer only, the results could be summed up in the statement that the pericardial organs in crabs (Cancer, Maia, Portunus) release a substance increasing the frequency and amplitude of the heart beat. Furthermore, the resemblance of the action of this substance to that of adrenaline and noradrenaline and the presence of a positive fluorescence reaction might lead to a conclusion that the function of the pericardial organs consists in liberating an adrenaline-like principle into the blood. The much weaker fluorescence reaction or its absence in the extracts from Maia would then show that the amount of this principle varies in different species of the Brachyura. From the experiments with the perfusion of the hearts in Homarus and. Squilla the same or a similar principle would be admitted in the Macrura and the Stomatopoda.

The reactions of the Maia heart are similar in that the amplitude of the beat is increased and a stabilizing effect can sometimes be observed in both species of crabs. There is, however, a remarkable discrepancy in the chronotropic action, for the extracts from both Cancer and Maia accelerate the heart of Cancer and slow that of Maia. As the heart of Maia is accelerated by adrenaline it must be assumed that either (i) the substance in the extracts which stimulates the heart of Cancer is not adrenaline-like, but an unknown principle of such properties that it excites the heart in one species and inhibits it in the other, or (ii) the same stimulating principle is present in both extracts but its effect on Maia heart is inhibited by the presence of some other factor to which this heart is more sensitive. If the second alternative is regarded more probable, two possibilities have to be considered: this second factor could be either some non-specific substance of the tissues of the pericardial organs extracted together with the stimulating principle, or it is another hormone which, in the living animal, is also released into the blood of the pericardial 
cavity. The negative results of the experiments with the extracts of various tissues do not favour the first alternative. On the other hand, the inhibiting effects on Maia heart of small amounts of this substance seem to point to a hormonal nature. If this is so we reach the conclusion that the pericardial organs in the Crustacea liberate two hormones, one stimulating and the other inhibiting the heart rhythm. To the latter can perhaps be ascribed the stabilizing effect recorded sometimes in Maia and Cancer. The different reactions exhibited by the hearts of the two species of Brachyura could be explained in this way: that the heart of Cancer is more sensitive to the action of the accelerator and that of Maia to the inhibitor; and, when treated by the artificial mixture of the two, each heart responds according to its particular sensitivity.

In the other crustaceans investigated (Homarus and Squilla) the pericardial organs can be assumed to release a stimulating hormone, but the evidence so far available does not permit us to draw any conclusion as to the presence or absence of the inhibitory hormone.

Although the idea that the pericardial organs may have a neurosecretory function was based on the histological evidence alone, the latter did not help much in the solution of the problem whether these organs produce two different substances. The fine neuropile-like terminations of the nerve fibres, by which presumably the substances are given off into the blood, do not show any differences. It is, however, worth noting that in Squilla the nerves forming the pericardial organs are without doubt made up of two different sets of fibres and, as has been suggested in the description of these organs (Alexandrowicz, I953a), their structure in the Decapoda may be the same, although here the diversity of origin of the nerve elements is not so easily discernible as in the Stomatopoda. If, therefore, it were proved that the pericardial organs in fact produced two hormones, the existence of two kinds of nerve fibres offers a possible explanation of their separate elaboration.

It appears from this work that in future physiological investigations of the crustacean heart the role of the pericardial organs must be taken into account. The experiments hitherto made on stimulation of the regulator cardiac nerves (for bibliography see Krijgsman, I952) were as a rule performed after the pericardium had been opened and the possibility of noting the action of the pericardial organs thus eliminated. Only Parrot (I94I) set up experiments in such a way that these organs could have produced their effect. This writer stimulated the inhibitory cardiac nerves in Maia in which the circulation was maintained, and took samples of the blood for testing on the intestine of the same animal. He found that such blood had a stimulating effect on the intestine and believed this to be caused by a substance released by the inhibitory nerves. This might have been so, but it seems more likely that in his experiments not only the inhibitory cardiac nerves but also the nerves of the pericardial organs were stimulated to release their hormones into the blood. 
Possibly also in the experiments of Wiersma \& Novitski (1942), in which the perfusion fluid was poured into the pericardial cavity, the pericardial organs had a chance to come into play; and conceivably their hormone was responsible for after-effects of inhibition supposed to be caused, as they wrote, by a 'liberation of a substance which can exert its influence at a later time'.

There seems to be no other instance when the action of the pericardial organs could be suspected of having affected the results of experiments (see the survey by Fredericq, 1947).

\section{SUMmary}

Experiments with isolated hearts of various Crustacea (Cancer pagurus, Maia squinado, Homarus vulgaris, Squilla mantis) were performed in order to test the postulated secretory function of the pericardial organs (nerve trunks of unusual structure lying in the pericardial cavity). Tests were made with extracts of these elements added to the fluid perfusing isolated hearts.

In Cancer, Homarus and Squilla the extracts caused a distinct increase in amplitude and frequency of the heart beat, and their effect proved to be very similar to that produced by adrenaline and noradrenaline tested on the same hearts. In Maia the extracts produced an increase in amplitude and decrease in frequency of the heart beat.

Extracts of the pericardial organs of Cancer pagurus gave the fluorescence reaction characteristic of adrenaline and related compounds. Blood taken from the pericardial cavity gave the same reaction, but that taken from the leg arteries did not.

It is assumed that the function of the pericardial organs in the Crustacea consists in liberating, through fine neuropile-like terminations of the nerve fibres, some hormone passing with the blood into the heart and producing on it a stimulating effect. There is certain evidence that the pericardial organs might release a second hormone having an inhibitory and perhaps also a stabilizing effect on the heart rhythm.

\section{REFERENCES}

AlexANDRowicz, J. S., I953 $a$. Nervous organs in the pericardial cavity of the decapod Crustacea. Fourn. Mar. Biol. Assoc., Vol. 31, pp. 563-80.

- $1953 b$. Notes on the nervous system in the Stomatopoda. II. The system of dorsal trunks. Pubbl. Staz. Zool. Napoli, Vol. 24, pp. 29-39.

BAIN, W. A., I929. The action of adrenaline and of certain drugs upon the isolated crustacean heart. Quart. Fourn. Exp. Physiol., Vol. 19, pp. 297-308.

DAVENPORT, D., I94I. The effects of acetylcholine, atropine, and nicotine on the isolated heart of the commercial crab, Cancer magister Dana. Physiol. Zool., Vol. I4, pp. I78-85.

FREDERICQ, H., I947. Les nerfs cardio-régulateurs des Invertébrés et la théorie des médiateurs chimiques. Biol. Rev., Vol. 22, pp. 297-3I4. 
GHIRETTI, F., I947. Azione dell'acetilcolina, dell'eserina, dell'atropina e dell' adrenalina sul cuore di un Crostaceo Decapodo: Eriphia spinifrons (Herbst). Pubbl. Staz. Zool. Napoli, Vol. 21, pp. IOI-9.

Hogben, L. T. \& Hobson, A. D., I924. Studies on internal secretion. III. The action of pituitary extract and adrenaline on contractile tissues of certain Invertebrata. Brit. Fourn. Exp. Biol., Vol. I, pp. 487-500.

KRIJGSMAN, B. J., I952. Contractile and pacemaker mechanisms of the heart of arthropods. Biol. Rev., Vol. 27, pp. 320-46.

PARROT, J. L., I94I.' Recherches sur la transmission chimique de l'influx nerveux chez les Crustacés. Libération d'une substance active sur l'intestin de Maia squinado par l'excitation des nerfs cardio-inhibiteurs. C.R. Soc. Biol., Paris, T. 135, pp. 929-33.

WelSH, J. H., I939a. Chemical mediation in crustaceans. I. The occurrence of acetylcholine in nervous tissues and its action on the decapod heart. Fourn. Exp. Biol., Vol. I6, pp. 198-219.

- I $939 \mathrm{~b}$. Chemical mediation on crustaceans. II. The action of acetylcholine and adrenalin on the isolated heart of Panulirus argus. Physiol. Zool., Vol.I2, pp. 23 I-7.

- 1942. Chemical mediation in crustaceans. IV. The action of acetylcholine on isolated hearts of Homarus and Carcinides. Fourn. Cell. Comp. Physiol., Vol. I9, pp. $27 \mathrm{I}-9$.

Wiersma, C. A. G. \& Novitski, E., I942. The mechanism of the nervous regulation of the crayfish heart. Fourn. Exp. Biol., Vol. 19, pp. 255-65. 